\title{
Abdominal cocoon: A rare cause of intestinal obstruction
}

\author{
M R Madan Karthik Raj
}

\begin{abstract}
Introduction: Abdominal cocoon syndrome is described as rare condition causing intestinal obstruction where a part or whole small bowel is encased in a fibrous membrane. Case Report: This case report is of a 30-year-old male patient who had undergone laparoscopic appendectomy five months ago presented with history, clinical and radiological findings suggestive of intestinal obstruction. He had been having similar episodes of intestinal obstruction which gets resolved spontaneously before and after surgery. Peroperatively inner aspect of entire peritoneal cavity was lined by a membrane which was adherent in certain areas causing obstruction. Adhesiolysis was done and patient recovered well. Conclusion: Awareness of this rare condition along with early diagnosis with radiological guidance and surgical intervention reduces morbidity of the patient.
\end{abstract}

Keywords: Intestinal obstruction, Abdominal cocoon

M R Madan Karthik Raj ${ }^{1}$

Affiliations: ${ }^{1} \mathrm{MS}$, Assistant Professor, Department of General Surgery, Vinayaka Mission's Kirupanandha variyar Medical College and Hospital, Salem, Tamil Nadu, India.

Corresponding Author: Dr. M R Madan Karthik Raj, MS (General Surgery), No 24 B, Shri saraswathi Apartments, Ramakrishna Road, Salem, Tamil Nadu, India; Postal Code: 636 007; Ph: 919894333979; E-mail: drmkraja@gmail.com

Received: 18 January 2013

Accepted: 10 May 2013

Published: 01 July 2013
Raj MRMK. Abdominal cocoon: A rare cause of intestinal obstruction. International Journal of Case Reports and Images 2013;4(7):345-348.

$$
* * * * * * * * *
$$

doi:10.5348/ijcri-2013-07-328-2

\section{INTRODUCTION}

Abdominal cocoon is a rare condition that refers to a total or partial encapsulation of the small bowel by a fibrocollagenous membrane or cocoon with local inflammatory reaction which leads to acute or chronic bowel obstruction. This was first described by Owtschinnikow in 1907 [1] as 'Peritonitis chronic fibrosa incapsulata' and as 'sclerosing encapsulating peritonitis' by Deeb et al. [2] in 1998. Abdominal cocoon usually presents as acute or recurrent sub acute intestinal obstruction with or without mass. Diagnosis of abdominal cocoon is usually made per operatively and surgical removal of obstruction causing membrane gives complete recovery. The aim of this report is to describe a young man with abdominal cocoon and to alert physicians to the diagnosis and management of this condition.

\section{CASE REPORT}

A 30-year-old male presented with vague complains of intermittent abdominal pain and back pain, nausea, vomiting and abdominal distension. He had undergone laparoscopic appendectomy five months ago. He had been having similar complaints before and after the surgery. No records about the operative findings in the previous surgery were available with the patient. On examination, his abdomen was distended with generalized tenderness in all quadrants. No free fluid in the abdomen made out on clinical examination. No mass or hernia was present in port sites. On 
auscultation, bowel sounds were sluggish. Initially the diagnosis was suspected as adhesive obstruction and was started on conservative management without symptomatic relief even after 12 hours. Blood counts were within normal limits. Computed tomography (CT) scan of abdomen showed all small bowel loops except duodenum and distal ileal loops were encased within a sac like structure with various thickness and small bowel showed signs of obstruction but no radiological evidence of mesenteric ischemia and suggested it to be a case of internal hernia or abdominal cocoon (Figure 1). Radiologist suggested patient to be a case of internal hernia or abdominal cocoon causing intestinal obstruction. Since patient did not respond well for conservative management, he was taken up for laparotomy. Per operatively thick membrane noted just after opening up the parietal peritoneum which had to be opened to visualize intestines. Inner aspect of entire peritoneal cavity, both parietal and visceral peritoneum was covered with a membrane of variable thickness and in some places membrane was adherent causing intestinal obstruction (Figure 2). Membrane causing obstruction were released with sharp dissection (Figure 3 ) and samples taken by peeling off the membrane from small bowel for biopsy (Figure 4). Since entire peritoneal cavity was covered with membrane, it could not be totally removed (Figure 5). Postoperative period was uneventful. Histopathological examination of the membrane showed nonspecific inflammatory cells with fibrosis.

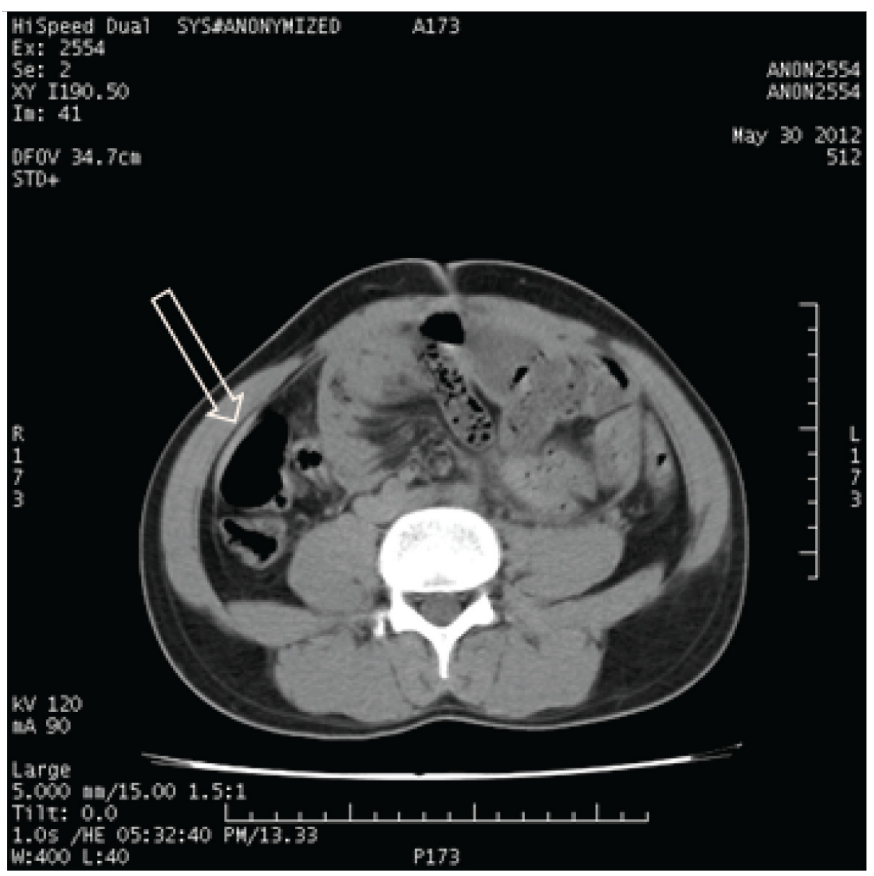

Figure 1: Computed tomography scan of abdomen. The arrow shows the membrane lining the peritoneum.

\section{DISCUSSION}

Abdominal cocoon, also referred to as sclerosing peritoneal encapsulation or sclerosing en-capsulating

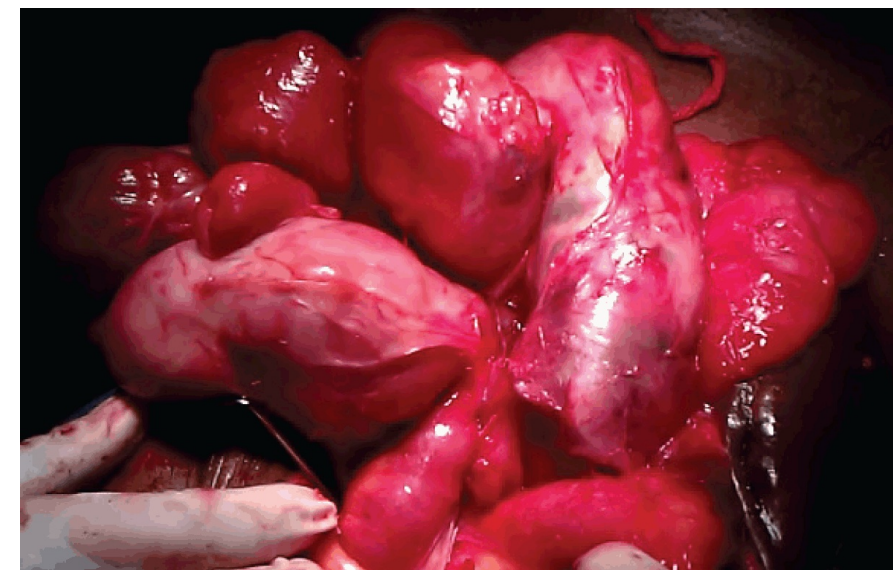

Figure 2: The appearance after opening the abdomen. Membrane covering the entire small bowel and area of adhesion causing obstruction can be seen.

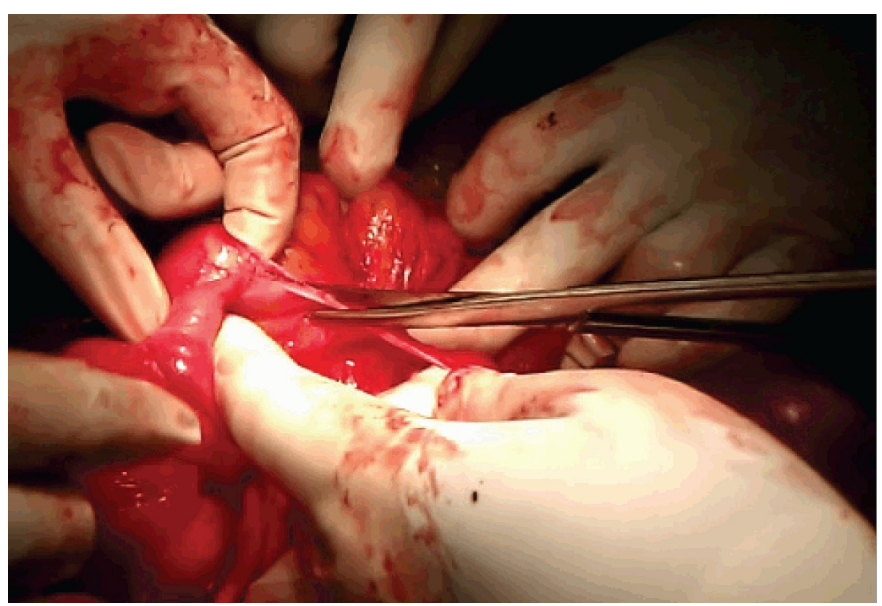

Figure 3: Release of adhesion by sharp dissection.

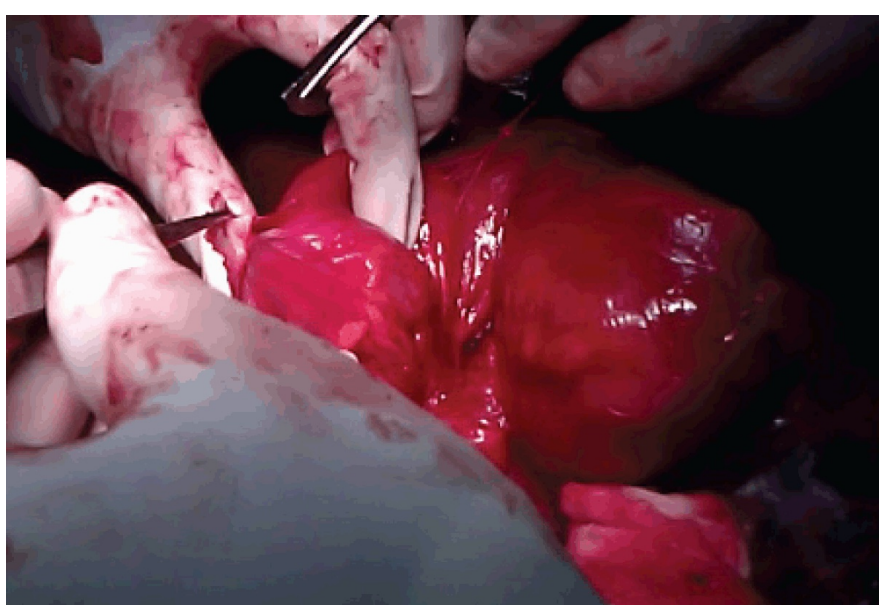

Figure 4: Peeling of the membrane covering the small bowel.

peritonitis, is a rare cause of acute abdomen, mostly involving young adolescent females. Abdominal cocoon may be classified into primary or idiopathic and secondary forms [3]. 


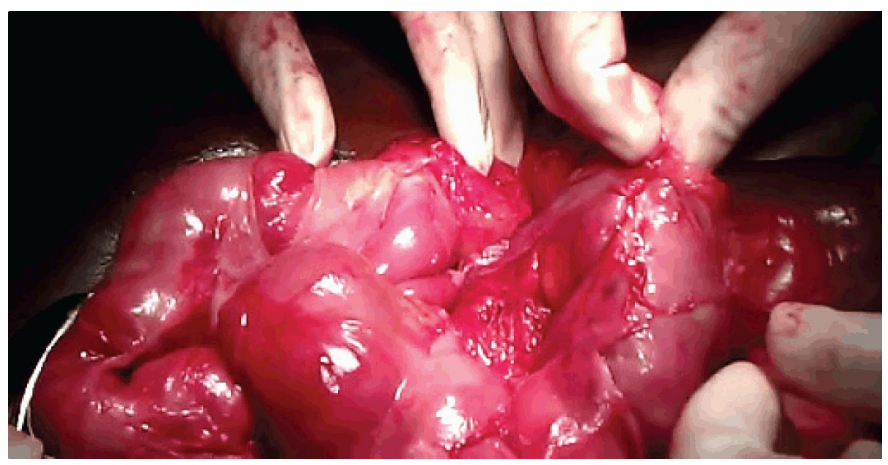

Figure 5: The appearance at the end of adhesiolysis. There is still membrane covering small bowel.

The pathogenesis of this syndrome remains unknown in primary type; however in secondary variant, etipathogenesis is co-related to congenital dysplasia, chronic asymptomatic peritonitis, some medicines (e.g., proctolol) continuous ambulatory peritoneal dialysis. Although retrograde menstruation with or without viral infection of the fallopian tubes has been suggested as a possible etiology [3], it does not account for the occasional occurrence of abdominal cocoon in males [4] as in our case. The clinical presentation of the abdominal cocoon syndrome mostly occurs as acute or sub acute intestinal obstruction which, in most cases, requires surgical intervention. Preoperative diagnosis of the syndrome is usually difficult. There are four main clinical features suggested in literature for preoperative diagnosis [5].

These are as follows:

(i) A relatively young female patient without an obvious cause of bowel obstruction.

(ii) Past history of similar episodes with symptomatic relief.

(iii) Presentation with symptoms suggestive of bowel obstruction but absence of cardinal symptoms such as distention.

(iv) Presence of soft non-tender abdominal mass.

However in our patient, similar episodes of intestinal obstruction with spontaneous symptomatic relief and abdominal distension were present. And the definitive diagnosis is achieved only after laparotomy. As described in literature, typically, the appearance of coil like pattern of the small bowel with areas of adhesion and dense thick membrane covering small and large bowel was seen [6]. The treatment of choice is adhesiolysis or total removal of membrane.

\section{CONCLUSION}

Abdominal cocoon syndrome is rare and difficult to diagnose condition which requires better awareness of the condition and a combination of clinical examination and radiologic studies may enhance chances of preoperative diagnosis. Early intervention and appropriate perioperative treatment can improve the outcome of this rare condition.

$$
* * * * * * * * *
$$

\section{Author Contributions}

M R Madan Karthik Raj - Substantial contributions to conception and design, Acquisition of data, Analysis and interpretation of data, Drafting the article, Revising it critically for important intellectual content, Final approval of the version to be published

\section{Guarantor}

The corresponding author is the guarantor of submission.

\section{Conflict of Interest}

Authors declare no conflict of interest.

\section{Copyright}

(C) M R Madan Karthik Raj 2013; This article is distributed under the terms of Creative Commons attribution 3.0 License which permits unrestricted use, distribution and reproduction in any means provided the original authors and original publisher are properly credited. (Please see www.ijcasereportsandimages.com /copyright-policy.php for more information.)

\section{REFERENCES}

1. Owtschinnikow PJ. Peritonitis chronic fibrosa incapsulata. ArchKlin Chir 1907;83:623-4. (Cited by): Constantinides F, DiNunno N, Bernasconi P, Jonjic N, Melato M. A new type of peritoneal encapsulation of the small bowel. Am J Surg Pathol 1998 Oct;22(10):1297-8.

2. Deeb LS, Mourad FH, El-Zein YR, Uthman SM. Abdominal cocoon in a man: preoperative diagnosis and literature review. J Clin Gastroenterol 1998;26(2):148-50.

3. Al-Abassi AA, Emad M. Abdominal cocoon. An unusual cause of intestinal obstruction. Saudi Med J 2004;25(10):1482-5.

4. Lalloo S, Krishna D, Maharajh J. Case report: abdominal cocoon associated with tuberculous pelvic inflammatory disease. $\mathrm{Br} \mathrm{J}$ Radiol 2002;75(890):174-6.

5. Yip FW, Lee SH. The abdominal cocoon. Aust N Z J Surg 1992;62(8):638-42.

6. Sieck JO, Cowgill R, Larkworthy W. Peritoneal encapsulation and abdominal cocoon. Case reports and a review of the literature. Gastroenterology 1983;84(6):1597-1601. 
Access PDF of article on other devices

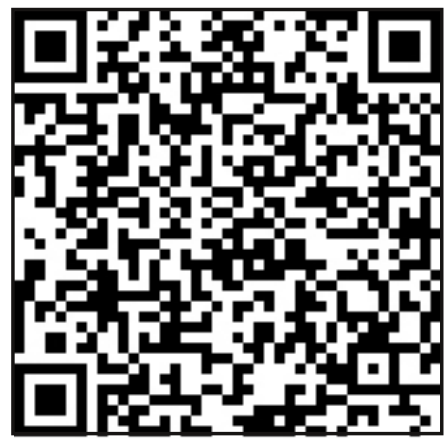

